\title{
On the variability of the accretion disk of AU Monocerotis
}

\author{
L. Celedón ${ }^{1}$, R.E. Mennickent ${ }^{1}$, S. Zharikov ${ }^{2}$, J.Garcés ${ }^{1}$, \\ M. Cabezas ${ }^{3}$ and G. Aguayo ${ }^{1}$ \\ 1 Departamento de Astronomía, Universidad de Concepción, Chile \\ 2 Instituto de Astronomía, Universidad Nacional Autónoma de México, \\ México \\ 3 Astronomical Institute of the Academy of Sciences of the Czech Republic, \\ Czech Republic
}

Received: October 15, 2019; Accepted: December 18, 2019

\begin{abstract}
AU Monocerotis is a well studied interacting binary star and a member of a group called Double Periodic Variables, whose principal characteristic is the existence of a long photometric cycle lasting on average about 33 times the orbital period. We made use of spectra covering from 2008 to 2015 to construct $\mathrm{H} \alpha$ Doppler maps. We found higher activity in the accretion disk during the low state of the system, similar to that found in HD 170582.
\end{abstract}

Key words: stars: binaries: eclipsing - stars: binaries: spectroscopic

\section{Introduction}

AU Monocerotis (GCRV 4526, HD 50846, HIP 33237) is an eclipsing interacting Algol-type binary with an orbital period of 11.113 days, and a member of the class called Double Periodic Variables (DPVs), with a long photometric cycle of 411 days (Lorenzi, 1985; Desmet et al., 2010; Mennickent, 2017). The system has shown a complex structure, with a permanent accretion disk and a transient high temperature accretion region, (Peters \& Polidan, 1984; Atwood-Stone et al., 2012) which have been revealed through spectroscopic studies.

Very few of the spectroscopic studies of DPVs take into account the high and low states of the long cycle separately. The most remarkable system in this sense is HD 170582, a Galactic DPV whose Doppler tomography in the low state shows higher disk emissivity with respect to the high state (Mennickent et al., 2016). This finding is consistent with the scenario proposed by Peters (1994) for AU Mon's long cycle, viz. mass transfer changes due to a variable radius of the donor star. 


\section{Data}

We acquired a total of 225 spectra between 2008 and 2015 in the visual range. The spectra were collected with 5 different telescopes and spectrographs: 172 at the San Pedro Martir (SPM) observatory, 39 with the CORALIE spectrograph, 7 with HARPS and 4 with the FEROS spectrograph, while 3 others were obtained with the echelle instrument at the DUPONT telescope. They were reduced and calibrated with IRAF. We separated them in two groups according to their long-cycle phase $\phi_{L}$ using the ephemerides given by Desmet et al. (2010): those with $\phi_{L}$ between 0.75 and 0.25 are referred to as high state and those with $\phi_{L}$ between 0.25 and 0.75 as low state. Most of the spectra fall in the high state of the long cycle (166 vs. 59), which is due to the high number of spectra taken at SPM during 2011 (86), covering almost an entire orbital cycle during the high state.

\section{Doppler tomography}

Doppler tomography is a technique that allows us to reconstruct the velocity space of the system from the available spectra in order the study the structure of the system (Marsh \& Horne, 1988).

For the construction of the Doppler maps we separated our data into three groups: the first one corresponds to the observations taken during 2011 with SPM (86), which cover almost an entire orbital cycle, the second one corresponds to the spectra catalogued as high state that are not part of the 2011 SPM observations (80), and the third group corresponds to the spectra catalogued as low state (59). For the creation of the Doppler maps we made use of the DTTVM code (Uemura et al., 2015).

The Doppler map of the 2011 SPM observations shows a structure fairly similar to the one observed by Atwood-Stone et al. (2012), with an accretion disk in emission, an emission source following the stream path and consistent with a hot-spot or the stream itself, a strong emission source in the fourth quadrant, similar to that observed in the map by Atwood-Stone et al. (2012), plus other emission zones between the third and fourth quadrants. Aside from the emission zones, we can observe a strong absorption bulge close to the primary star, which can be interpreted as evidence for the impact of the stream on the surface of the star (Richards et al., 2014).

The maps of the high and low state show a similar structure. However, we observe a difference in the emissivity of both maps, with the low state showing a greater emissivity in the first quadrant with respect to the high state. The rest of structures show a similar behaviour between both maps.

To check if this behaviour is due to data sampling, we created synthetic spectra of an accretion disk using the code Shellspec3.9 (Budaj \& Richards, 2004). For the parameters of the disk we used the physical parameters reported 
by Atwood-Stone et al. (2012). We emulated the orbital phases of both sets of data, high and low, and then created a Doppler map for the synthetic spectra. The resulting maps do not show a strong emissivity during the low states, suggesting that the higher emissivity during the low state observed in the real Doppler maps is intrinsic to the system.

\section{Conclusions and discussion}

For the first time we performed Doppler tomography for AU Mon in its high and low states, finding a strong emission feature at the first quadrant during the low state of the cycle.
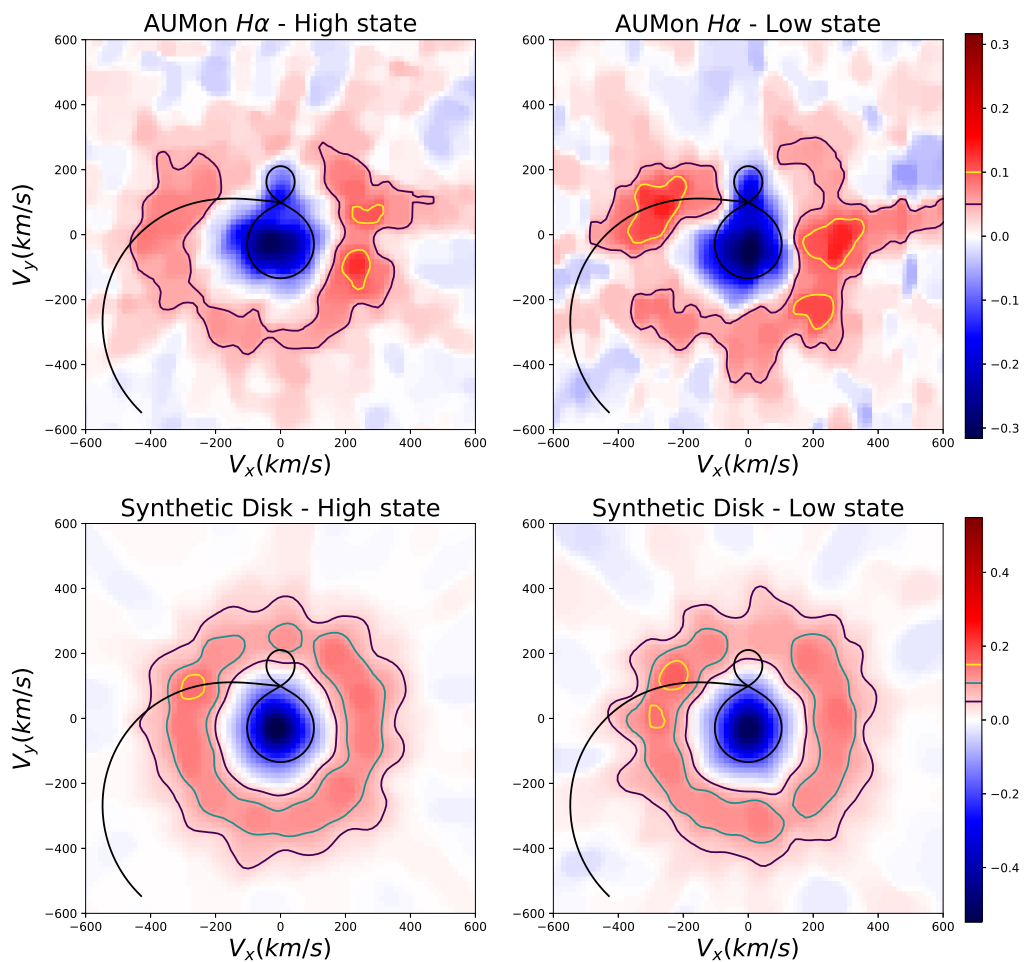

Figure 1. Doppler tomography for AU Mon in $\mathrm{H} \alpha$ in High and Low state (up) and for the synthetic accretion disk (bottom). The contour lines indicate levels of emission of $0.05,0.1$ and 0.15 above the continuum level.

This behaviour is also observed in HD 170582, and it can be interpreted as evidence of an optically thinner disk during the low state of the long cycle. This is consistent with the scenario of variable mass transfer proposed by Peters 
(1994) for AU Mon. Eventually, we may expand this model not only for AU Mon, but for DPVs in general, where the long cycle should be produced by this variable mass transfer due to a variation of the radius of the secondary star. This scenario is further developed in the dynamo model by Schleicher \& Mennickent (2017).

Acknowledgements. The authors are grateful to the CONICYT Programa de Astronoma Fondo ALMA-CONICYT 201731170002 and Fondecyt 1190621.

\section{References}

Atwood-Stone, C., Miller, B. P., Richards, M. T., Budaj, J., \& Peters, G. J., Modeling the Accretion Structure of AU Mon. 2012, Astrophys. J., 760, 134, DOI: 10.1088/0004-637X/760/2/134

Budaj, J. \& Richards, M. T., A description of the shellspec code. 2004, Contributions of the Astronomical Observatory Skalnate Pleso, 34, 167

Desmet, M., Frémat, Y., Baudin, F., et al., CoRoT photometry and high-resolution spectroscopy of the interacting eclipsing binary AU Monocerotis. 2010, Mon. Not. R. Astron. Soc., 401, 418, DOI: 10.1111/j.1365-2966.2009.15659.x

Lorenzi, L., Confirmation of the Regular Intrinsic Variability of AU Monocerotis. 1985, Information Bulletin on Variable Stars, 2704, 1

Marsh, T. R. \& Horne, K., Images of accretion discs - II. Doppler tomography. 1988, Mon. Not. R. Astron. Soc., 235, 269, DOI: 10.1093/mnras/235.1.269

Mennickent, R. E., Long Photometric Cycles in Hot Algols. 2017, Serbian Astronomical Journal, 194, 1, DOI: 10.2298/SAJ1794001M

Mennickent, R. E., Zharikov, S., Cabezas, M., \& Djurašević, G., Doppler tomography of the Double periodic variable HD 170582 at low and high stage. 2016, Mon. Not. R. Astron. Soc., 461, 1674, DOI: 10.1093/mnras/stw1416

Peters, G. J., The Nature of the Periodic, Long-Term Variations in the Circumstellar Material in AU Monocerotis. 1994, in Astronomical Society of the Pacific Conference Series, Vol. 56, Interacting Binary Stars, ed. A. W. Shafter, 384

Peters, G. J. \& Polidan, R. S., Evidence for a high-temperature accretion region in Algol-type binarysystems. 1984, Astrophys. J., 283, 745, DOI: 10.1086/162359

Richards, M. T., Cocking, A. S., Fisher, J. G., \& Conover, M. J., Images of Gravitational and Magnetic Phenomena Derived from Two-dimensional Back-projection Doppler Tomography of Interacting Binary Stars. 2014, Astrophys. J., 795, 160, DOI: $10.1088 / 0004-637 \mathrm{X} / 795 / 2 / 160$

Schleicher, D. R. G. \& Mennickent, R. E., A dynamo mechanism as the potential origin of the long cycle in double periodic variables. 2017, Astron. Astrophys., 602, A109, DOI: $10.1051 / 0004-6361 / 201628900$

Uemura, M., Kato, T., Nogami, D., \& Mennickent, R., Doppler tomography by total variation minimization. 2015, Publ. Astron. Soc. Jap., 67, 22, DOI: $10.1093 / \mathrm{pasj} / \mathrm{psu} 154$ 